\title{
Capacidad de absorción: aproximaciones teóricas y empíricas para el sector servicios
}

\author{
Aguilar-Olaves, Gabriel* \\ Herrera, Liliana** \\ Clemenza, Caterina ${ }^{\star \star \star}$
}

\section{Resumen}

En este estudio se analizaron los determinantes de la capacidad de absorción en el sector servicios y el poder explicativo de la amplia heterogeneidad que presenta la actividad innovadora de las empresas de este sector. Se empleó un análisis factorial exploratorio, que agrupó los determinantes de la capacidad de absorción en dos dimensiones: capacidades potenciales y capacidades realizables, y un análisis discriminante para establecer, a partir de estas dimensiones, qué diferencias hay entre las empresas de este sector. Los resultados sugieren que estas dimensiones pueden ser cuantificables y explicarían las diferencias significativas en el gasto en innovación y el tipo de innovación que obtienen las empresas del sector servicios. Adicionalmente el estudio concluye, para el caso analizado, que el gasto en innovación no es siempre un elemento determinante de la capacidad de absorción y que la innovación en el sector servicios estaría principalmente orientada al desarrollo de cambios organizativos y de procesos a diferencia de introducir nuevos productos para el mercado.

Palabras clave: Capacidad de absorción, proceso innovador, sector servicios, gasto en innovación, innovaciones radicales, innovaciones incrementales.

\section{Recibido: 30-04-12. Aceptado: 26-09-13}

* Economista. Magíster en Gerencia de Operaciones. Magíster en Investigación y Economía de la Empresa. Especialista en Gerencia de Proyectos. Investigador del Instituto de Investigaciones de la Facultad de Ciencias Económicas y Sociales. Universidad del Zulia. Venezuela. Investigador del Departamento de Dirección y Economía de la Empresa. Universidad de León. León, España, e-mail: gaguo@unileon.es.

** Doctora en Dirección y Economía de la Empresa. Profesora Contratada. Departamento de Dirección y Economía de la Empresa. Universidad de León. León, España, e-mail: liliana.herrera@unileon.es.

*** Doctora en Ciencias Gerenciales. Post-doctora en Ciencias de la Educación. Profesora-investigadora Titular adscrita al Instituto de Investigaciones de la Facultad de Ciencias Económicas y Sociales de la Universidad del Zulia. Acreditada al Programa de Estímulo a la Investigación (PEI-ONCTI), e-mail: caterinaclemenza@yahoo.es. 


\title{
Capacity for Absorption: Theoretical and Empirical Approaches to the Service Sector
}

\begin{abstract}
In this study, the determinants for absorption capacity in the service sector and the explanatory power of the broad heterogeneity presented by the innovative activity of businesses in this sector were analyzed. An exploratory factorial analysis was used, which grouped the determinants for adsorption capacity into two dimensions: potential capacities and realizable capacities; a discriminant analysis was used to establish, based on these dimensions, the differences that exist among businesses in this sector. The results suggest that these dimensions can be quantifiable and could explain significant differences in the expenditure for innovation and the type of innovation that businesses in the service sector obtain. Additionally, the study concludes that for the case analyzed, the expenditure for innovation is not always a determining element for the capacity of absorption and that innovation in the service sector would be principally oriented to developing organizational changes and processes, instead of introducing new products to the market.
\end{abstract}

Key words: Absorption capacity, innovating process, service sector, innovation expense, radial innovations, incremental innovations.

\section{Introducción}

En los últimos años ha crecido el interés por el estudio de la innovación en el sector servicios gracias al reconocimiento general de su aportación al Producto Interno Bruto (PIB) y el empleo y su implicación en el desarrollo económico, científico y social. La existencia del sector servicios no solo complementa el desempeño del sector manufacturero, sino que sistemáticamente promueve una espiral de crecimiento de este último.

Los estudios sobre innovación hasta ahora realizados, se han enfocado en analizar la intensidad del conocimiento en el sector servicios (Amara et al., 2009) con especial énfasis en los servicios comerciales y financieros (Miles et al., 1995). Sin embargo, en la literatura hay todavía una notoria falta de abstracción en el estudio del comportamiento innova- dor del sector servicios llegando a no revelarse de forma concreta y precisa cómo se produce la innovación en este sector, cuales son las consecuencias de la innovación en el desempeño y rendimiento de las empresas o, más importante aún, cómo las empresas en este sector se aprovisionan de capacidades internas para innovar o asimilar conocimiento de su entorno.

En este estudio se profundiza en el estudio de la capacidad de absorción (Absorptive Capacity, ACAP, en sus siglas en inglés) en el sector servicios como aspecto clave para el desarrollo del proceso innovador. ACAP es definida por Cohen y Levinthal (1989) como la habilidad de una empresa para organizar, identificar, asimilar y explotar el conocimiento proveniente del exterior. El concepto, se fundamenta en la visón de que el conocimiento interno no es suficiente y 
las empresas necesitan fuentes de conocimiento externo para mejorar los resultados del proceso innovador. En los últimos años el concepto ha alcanzado especial importancia al ser considerado un factor determinante de la conducta innovadora y un factor clave del éxito empresarial. En general, el concepto de ACAP se ha utilizado ampliamente en el estudio del comportamiento innovador del sector manufacturero y su aplicación en el sector servicio es prácticamente inexistente, debido, en parte, a las dificultades para encontrar medidas que permitan operacionalizar el desarrollo conceptual. El estudio de Zahra y George (2002) advierte de la existencia de serías limitaciones en la medición de ACAP, así como de ambigüedades en su definición y variedad de interpretaciones. Mientras que en algunos estudios se le ha atribuido una naturaleza cualitativa (Murovec y Prodan, 2009), en otros se señala que la presencia de asociaciones lineales entre variables explicativas de esta capacidad dificulta la medición cuantitativa del concepto (Tsai, 2009). En la actualidad todavía existe un amplio debate sobre la medida más adecuada y precisa para cuantificar e identificar ACAP en las empresas, sin embargo esta es clave para el desarrollo de la actividad innovadora de las empresas, por tanto se hace necesario avanzar en el estudio de su medición.

En este contexto, este trabajo tiene dos objetivos. Primero, identificar los factores que componen o determinan ACAP en el sector servicios y, segundo, comprobar su poder explicativo para discriminar a las empresas del sector servicios en términos de las entradas y salidas del proceso innovador. Para ello, se emplea- rá un análisis factorial de componentes principales, utilizando una serie de variables analizadas en investigaciones previas que indican que la ACAP se compone de distintas habilidades para adquirir conocimiento, asimilarlo, transformarlo y explotarlo. En el estudio se ha realizado una amplia revisión de la literatura que evalúa estos factores a través de técnicas de medidas cualitativas y cuantitativas, entre las que se pueden mencionar las desarrolladas por: Jansen et al. (2005); Khoja y Maranville (2010); Jiménez-Barrionuevo et al. (2011); Nieto y Quevedo (2005); (para las medidas cualitativas); y Cohen y Levinthal (1990); Kim (1980); Mowery et al. (1996); Kim (1999); Van den Bosch et al. (1999); Volberda et al. (2010); Todorova y Durisin (2007); Newey y Shulman (2004) (para la medición cuantitativa).

A continuación, una vez obtenida las medidas para las dimensiones de ACAP en el sector servicios (objetivo 1), se emplea un análisis discriminante para determinar de qué manera estas dimensiones explicarían las diferencias significativas entre las empresas del sector en cuanto a entradas (gasto en innovación) y salidas del proceso innovador (tipo de innovación y su grado de novedad de la innovación) del proceso innovador (objetivo 2). Como aportación de esta investigación se emplea una fuente de datos cuantitativa que abarca un amplio rango de empresa del sector servicios, por lo cual se podrá obtener una visión general de los factores que componen la ACAP en este sector y su influencia en la actividad innovadora.

El trabajo se estructura como sigue. En la segunda sección se presenta 
el marco teórico y las hipótesis del estudio. En la tercera se exponen los aspectos metodológicos y en la cuarta se describen y discuten los resultados del análisis empírico. Finalmente en la quinta se presentan las conclusiones.

\section{Capacidad de absorción en las empresas}

Originalmente la ACAP es definida como la habilidad de las organizaciones para explotar conocimiento externo (Cohen y Levinthal, 1990). Estudios recientes le han asignado un papel más determinante en la actividad innovadora que pasa por reconocer que la ACAP no sólo contribuye a reconocer el valor del conocimiento externo, sino también facilita su asimilación, aplicación y uso comercial (Escribano et al., 2009). Para llegar a ello, las empresas pasan por un proceso secuencial en el que 1) reconoce el conocimiento potencialmente valioso para la empresa (aprendizaje explorador) 2) se asimila ese nuevo conocimiento (aprendizaje transformador) y, 3) se utiliza para crear nuevo conocimiento con valor de mercado (aprendizaje explotador).

En definitiva, la ACAP es el resultado de un prolongado proceso de inversión y acumulación de conocimientos dentro de la organización que se ve influenciado por la participación de la empresa en mercados de productos específicos, las actividades de investigación y desarrollo $(I+D)$ y el progreso de su trayectoria en el pasado (path-dependecy). Zahra y George (2002) le asignan un rol más organizacional definiéndola como un conjunto de rutinas y procesos orga- nizativos de aprendizaje y creación de conocimiento.

\subsection{Capacidades potenciales y realizables en las empresas}

La visión más actual de ACAP la define como una capacidad dinámica (Zahra y George, 2002) lo que ha permitido relacionarla con la habilidad de la empresa para alcanzar una ventaja competitiva sostenible (Zahra et al., 2006; Todorova y Durisin, 2007; Narasimhan et al., 2006). De acuerdo con Teece et al., 1997), las capacidades dinámicas son habilidades que tienen las empresas para lograr nuevas formas de obtener ventajas competitivas, partiendo de la integración, construcción y re-configuración de competencias internas y externas que le facilitan adaptarse a los cambios en el entorno. Estudios recientes reconocen en estas capacidades un valor estratégico (Mowery et al., 1996) y las vinculan con la habilidad de las empresas para crear no solo nuevos productos y servicios, sino también una mejor posición competitiva en el mercado (Dunning y Lundan, 2010). A pesar de la importancia atribuida a estas capacidades dinámicas en el proceso innovador, no hay consenso en su definición o en cómo deberían ser operacionalizadas (Di Stefano et al., 2010).

En el caso concreto de ACAP, los estudios de Zahra y George (2002) y Todorova y Durisin (2007) han avanzado mucho en la aproximación conceptual de esta capacidad. De acuerdo con estos autores, la ACAP estaría compuesta de cuatro rutinas y procesos organizativos que comprenden la adquisición, asimilación, trans- 
formación y explotación el conocimiento. Zahra y George (2002)agrupan éstas cuatro dimensiones en dos grandes bloques denominados Capacidad de Absorción Potencial (PACAP) y Capacidad de Absorción Realizable (RACAP).

Mientras que la RACAP reúne la transformación y explotación del conocimiento, la PACAP agrupa la adquisición y asimilación del conocimiento. Estos dos grupos tienen un distinto valor estratégico para las empresas. La PACAP permite que la organización pueda adaptarse de forma más eficiente a los cambios del entorno y el mercado, interiorizando y asimilando el conocimiento. La RACAP influye en el rendimiento de la organización mediante la transformación y explotación del conocimiento que da lugar a la innovación de productos y/o procesos.

Aunque la PACAP y la RACAP resultan ser dimensiones esenciales para explicar la ACAP, Zahra y George (2002) y Murovec y Prodan (2009) advierten sobre las ambigüedades existentes en su definición e interpretación, que subyacen en su naturaleza cualitativa, y dificultan encontrar adecuadas medidas cuantitativas para el análisis. Desde la perspectiva cuantitativa, Tsai (2009) señala la colinealidad existente entre la ACAP (medida por el cociente entre gasto en I+D y las ventas, o gasto en formación y personal en $\mathrm{I}+\mathrm{D}$ ) y otras variables explicativas o de control.

No obstante, estudios recientes han hecho un intento por mejorar estas medidas de ACAP (Arbussa y Coenders, 2007; Murovec y Prodan, 2009). De ahí que tras una revisión de la literatura se propone una medición cuantitativa de ACAP basada en estudios que la han tratado como variable dependiente e independiente en sus análisis. El Cuadro 1 muestra los estudios analizados, clasificados ellos de acuerdo con las cuatro dimensiones de la ACAP y la naturaleza del estudio (cuantitativa vs. cualitativa).

La PACAP es definida por Zahra y George (2002) como prácticas que fo-

\section{Cuadro 1 \\ ACAP: Relación entre estudios con mediciones cualitativas y cuantitativas}

\begin{tabular}{|c|c|c|c|}
\hline & Dimensiones & Medición Cualitativa & Medición Cuantitativa \\
\hline PACAP & Adquisición & $\begin{array}{l}\text { Jansen et al., 2005; Khoja y } \\
\text { Maranville, 2010;Jiménez- } \\
\text { Barrionuevo et al., 2011; Nie- } \\
\text { to y Quevedo, 2005; Rush et } \\
\text { al., } 2007 .\end{array}$ & $\begin{array}{l}\text { Cohen y Levinthal, 1990; Kim, } \\
\text { 1980; Mowery et al., 1996; Kim, } \\
\text { 1999; Van den Bosch et al., } \\
\text { 1999; Volberda et al., 2010; To- } \\
\text { dorova y Durisin, 2007; Newey y } \\
\text { Shulman, } 2004 .\end{array}$ \\
\hline \multicolumn{4}{|c|}{ Asimilación } \\
\hline RACAP & Transformación & $\begin{array}{l}\text { Jansen et al., 2005;Jimé- } \\
\text { nez-Barrionuevo et al., 2010; } \\
\text { Nieto y Quevedo, 2005; Jan- } \\
\text { tunen et al., 2005. }\end{array}$ & $\begin{array}{l}\text { Cohen y Levinthal, 1990; Kim, } \\
\text { 1999; Volberda et al., 2010; } \\
\text { Szulanski, 1996; Newey y Shul- } \\
\text { man, 2004; Kim, } 1980 .\end{array}$ \\
\hline
\end{tabular}

Fuente: Elaboración propia. 
mentan medios de comunicación con el entorno y el establecimiento de redes de trabajo. Estas prácticas son determinantes de la innovación y contribuyen significativamente al desarrollo de la capacidad innovadora de la empresa, permitiendo a través de la exposición a nuevas fuentes de ideas (Tether y Tajar, 2008), el rápido acceso a recursos y la transferencia de conocimiento (Caloghirou et al., 2004).

De acuerdo con Kostopoulos et al. (2011), el acceso a fuentes externas de conocimiento, permite a la organización tomar ventaja en dos aspectos que son críticos. Por un lado, ganar acceso a nuevas fuentes de conocimientos y habilidades, y por otro, desarrollar la capacidad para interpretar e incorporar este nuevo conocimiento a la base de la empresa, quienes acceden a él mediante distintas fuentes como: proveedores de materia prima y suministro, usuarios, universidades, institutos de investigación o incluso competidores. El establecimiento de un mecanismo de intercambio y/o transferencia de conocimiento con el exterior, asume un lugar privilegiado en el desarrollo de habilidades de una organización (Laney y Lubatkin, 1998).

La RACAP, la define Zahra y George (2002) como habilidades que se extienden y apalancan en las competencias previas existentes, o que bien se van generando a medida que se van desarrollando las actividades rutinarias de la empresa. La RACAP permite que la empresa reconozca diferentes conjuntos de información, aparentemente incongruentes, y los convierta en nuevo conocimiento. También permite a la empresa realizar todas aquellas actividades de refinación, extensión y apalancamiento de las rutinas, que le ayudarían a explotar su potencial y capacidades. Para Todorova y Duri$\sin$ (2007) la RACAP puede fomentar y fortalecer las relaciones hacia el interior de la organización, así como hacia el exterior, con consumidores y demás interesados (stakeholders), ya que desarrolla y refina las rutinas organizativas combinando el conocimiento existente y el recientemente adquirido y asimilado.

Si bien, la PACAP y la RACAP, definidas previamente por Zahra y George son los pilares fundamentales de la ACAP, se requiere del cumplimiento de dos premisas para alcanzar dicha capacidad (Cohen y Levinthal, 1990; Kim, 1999). Primero, que exista conocimiento previo para evaluar y que se utilicen fuentes externas de conocimiento. Esto supone la existencia de un componente tácito que permita que la entrada de conocimiento a la organización fluya de forma eficiente (Mowery et al., 1996). Segundo, debe producirse un esfuerzo innovador, que fomente intercambios de conocimiento entre los miembros de la organización (Escribano et al., 2009). En términos cuantitativos, la existencia de gastos en innovación en la empresa (aquellos dirigidos a la búsqueda de conocimiento) es una medida de aproximación (variable proxy) del cumplimiento de estas premisas. Como resultado se espera que siendo la PACAP y la RACAP elementos determinantes de la innovación, permitan evaluar los perfiles de las empresas del sector servicios en función del gasto en innovación.

En función a lo anterior, se plantean dos hipótesis de investigación: 
Hipótesis 1. La PACAP y la RACAP determinan las diferencias en el gasto de innovación y el perfil entre las empresas del sector servicios.

Si bien, la estructura de ACAP puede ser determinante de una entrada del proceso innovador como lo es el gasto en innovación, puede también ser clave en la introducción de nuevos productos y servicios, o en la mejora de los ya existentes. De acuerdo con Kim (1980) estas salidas del proceso de innovación (innovaciones radicales e innovaciones incrementales) de cara a lograr una mayor competitividad, deben sustentarse en la implementación, la asimilación, la transformación y la explotación del conocimiento, con lo que se alcanzaría una mayor capacidad para la solución de problemas. En este contexto, la PACAP y la RACAP son determinantes no solo para crear innovación, sino también una ventaja competitiva sostenible (Zahra y George, 2002). Como resultado, parece existir una relación directa entre la composición de ACAP y los resultados del proceso innovador. Esas dimensiones pueden ser claves en distintos tipos de resultados innovadores y pueden ser un elemento discriminante entre empresas con resultados innovadores y sin ellos. Por tal razón se formula la siguiente hipótesis.

Hipótesis 2. La PACAP y RACAP determinan las diferencias en los resultados innovadores y el perfil entre las empresas del sector servicios.

Las hipótesis formuladas en este estudio se contrastan en una muestra de empresas del sector servicios para determinar, entre otros, si la estructura teórica de ACAP propuesta por Zara y George (2002) es aplicable a este sector. De serlo, se espera que las dimensiones identificadas puedan explicar las entradas y salidas del proceso innovador en este sector.

\section{Medidas de la ACAP en el sector servicios}

El interés por la innovación en el sector servicios recientemente ha tenido una importancia sin precedentes, a un nivel tal que, algunos organismos como la Comisión Europea la han catalogado como una de las líneas estratégicas prioritarias de desarrollo para los países de la zona euro. Uno de los países que más ha manifestado su interés en analizar las relaciones entre los servicios, la oferta tecnológica y la promoción de las políticas públicas para la innovación ha sido España; quien se sitúa el séptimo lugar entre los países de la Unión Europea con mayor gasto interno en $\mathrm{I}+\mathrm{D}$ en relación al PIB, y cuya media está por encima de la del grupo de 27 países de la Unión Europea (Informe COTEC, 2009). El ritmo del esfuerzo innovador en los últimos años se ha mantenido en cifras que superan las tasas de crecimiento del PIB y ha propiciado el aumento de la inversión en I+D en comunidades autónomas como Navarra, País Vasco, Madrid, Cataluña, Aragón Cantabria y Castilla y León, Andalucía y la Comunidad Valenciana.

En tal sentido, el análisis empírico de este trabajo se llevó a cabo con datos procedentes de la encuesta de Panel de 
Innovación Tecnológica (PITEC) ${ }^{1}$ en España, para el periodo 2008. Aunque actualmente hay datos disponibles hasta el año 2011, la actualización de esta base de datos no es regular y la investigación no ha podido ir al ritmo de la actualización. Por otro lado, se ha seleccionado el año 2008 como año de referencia, por ser este el año anterior al comienzo de la crisis española. Los datos correspondientes a los años 2009-2011 disponen de un $35 \%$ menos de empresas innovadoras en la muestra.

La investigación utilizó información de 5099 empresas que de acuerdo con la Clasificación Nacional de Actividades Económica 2003 (CNAE, 2003²) corresponden al sector de servicios en las categorías: comercio al por mayor y al por menor, transporte y almacenamiento, hostelería, información y comunicaciones, actividades financieras, actividades inmobiliarias, actividades profesionales, administración pública y defensa, educación, actividades artísticas y otros servicios; que incluyen todos ellos empresas que realizaron gastos en $\mathrm{I}+\mathrm{D}$ y obtuvieron resultados innovadores, como aquellas que no.

La metodología empleada está sustentada en la utilización de técnicas multivariantes $^{3}$, las cuales permiten cuantificar mejor las diferencias en los perfiles y el esfuerzo innovador de las empresas del sector servicios. Para el contraste de las hipótesis fue necesario obtener una estructura de ACAP. Para este propósito se utilizó un análisis factorial con el fin de explorar y definir la estructura subyacente de los componentes de la ACAP en este sector. Se analizan las interrelaciones entre las variables y se definen una serie de dimensiones subyacente comunes entre sí, describiendo con ello el fenómeno de una manera más sintetizada que con las variables originales. El Cuadro 2 recoge las medidas de las variables empleadas en este análisis, seleccionadas en función de la revisión de la literatura y dada la naturaleza multivariante del constructo ACAP. El anexo 1 se puede consultar para ver el nombre completo de cada etiqueta de variable.

Una vez obtenida la medida de la ACAP, se construyeron 2 modelos de análisis discriminante para establecer las diferencias y el perfil de las empresas del sector servicios en cuanto al gasto en innovación (entrada) y el resultado innovador (salida). Las estimaciones de los modelos se realizaron mediante el software estadístico STATA 10.1 y se empleó un análisis por etapas para excluir del modelo variables no significativas. En cada modelo se realizan dos tipos de análisis. El primero, arroja coeficientes que permiten

1 Para ver más sobre la encuesta de Panel de Innovación Tecnológica visitar: http://icono. fecyt.es/PITEC/Paginas/descarga_bbdd.aspx

2 Para ampliar mas sobre la clasificación de actividades, visitar: http://www.ine.es/inebmenu/mnu_clasifica.htm

3 Para ampliar sobre las técnicas multivariantes de análisis de factorial y análisis discriminantes ver Hair et al., (1999 


\section{Cuadro 2 \\ Definición de Variables para la medición de la ACAP}

\section{Etiqueta}

Innovación en bienes, Innovación en servicios, Innova-

ción en productos

Fuentes externas: Universi- Variables dicotómicas relacionadas con las fuentes de dades $u$ otros centros de en- información a las que han tenido acceso las empresas. señanza superior, Centros Estas variables se clasifican en Fuentes de Información tecnológicos, Comerciales y Institucionales (universidades, centros tecnológicos), de Mercado (consultores, la- Fuentes de Información del Mercado (competidores, boratorios comerciales pri- consultores y laboratorios comerciales de I+D).

vados de $I+D$ )

Innovaciones en las relaciones con otras empresas.

Innovaciones en los sistemas de gestión de los conocimientos.

Innovaciones en las estructuras de gestión e integración de los departamentos.$$
\begin{aligned}
& \text { innovaciones en la organización de los lugares de trabajo } \\
& \text { de la empresa, reparto de responsabilidades y toma de } \\
& \text { decisiones. }
\end{aligned}
$$

Innovaciones en nuevos mé- Variable dicotómica que se define como innovación cotodos de comercialización mercial, y supone la incorporación de nuevas estrate-

Importancia del efecto sobre: capacidad de producción o prestación de servicios, costos laborales por unidad producida, energía por unidad producida, flexibilidad en la producción o prestación de servicios, materiales por unidad producida, calidad de los productos y servicios, cuota de mercado, penetración de nuevos mercados, sustitución de productos y servicios, impacto salud y seguridad, impacto en el medio ambiente, cumplimiento de los requisitos normativos.

Incentivos y retribuciones a investigadores, técnicos auxiliares $\mathrm{y} / \mathrm{o}$ becarios gias o nuevos métodos comerciales que conllevan al posicionamiento del producto en el mercado.

Estas variables recogen información sobre la aplicación de nuevos métodos organizativos para el desarrollo de actividades internas de la empresa. Las variables son dicotómicas y especifican innovaciones organizativas para el desempeño de las relaciones externas con otra empresa, innovaciones organizativas en la gestión de la organización del trabajo y procedimientos de la empresa, e Estas variable, medidas en 1,0; recogen información de los objetivos que persigue la empresa a través de la actividad innovadora, orientada a: Procesos (mayor capacidad de producción o prestación de servicio, menores costes laborales, menos consumo de energía, mayor flexibilidad en el sistema de producción o prestación de servicio, menos consumo de materiales), Productos (mayor calidad de los bienes y servicios, mayor cuota de mercado, penetración en nuevos mercados, sustitución de productos o procesos anticuados) y Seguridad y Medioambiente (menor impacto medioambiental, mejora en la salud y la seguridad, cumplimiento de requisitos normativos medioambientales.
Informa sobre la retribuciones hechas a investigadores, becarios, técnicos, auxiliares y otros gastos corrientes relacionados con las actividades de I+D. Medida 1,0 .

Fuente: Elaboración propia.

Chen, 2004;

Ziggers y Henseler, 2009;

Murovec y Prodan, 2009;

Jiménez-Barrionuevo et

al., 2010.

Ortega, 2010. 
establecer si las variables utilizadas explican las diferencias entre las empresas del sector servicios en función de las entradas y salidas del proceso de innovación, y el segundo determina cuales de esas variables independientes tienen mayor importancia relativa en función de su coeficiente en el modelo. En este último análisis se presenta información de 2 grupos de empresas: aquellas que realizan gasto en innovación (entrada) u obtuvieron resultado innovador (salida), frente aquellas que no. Como resultado, se entiende que una variable tiene importancia relativa en el modelo si su valor medio es más alto en un grupo que en otro.

La definición que la literatura hace sobre las variables que influyen en la ACAP, así como su demostrada utilización en diversos estudios y aporte significativo en la explicación de dicho fenómeno, sustentan la cuidadosa selección de las variables dependientes e independientes.

En el caso de las variables dependientes, la primera es una entrada del proceso de la innovación que corresponde al gasto en innovación que realizan las organizaciones. Esta variable ofrece la opción de poder agrupar aquellas empresas que: 1) han realizado gasto interno y externo en I+D, adquisición de I+D, adquisición de maquinarias equipos o software, y 2) han realizado gasto en formación y capacitación de personal. La segunda y tercera variables, son salidas 0 resultados del proceso innovador definidas como innovación radical e innovación incremental. La primera de estas recoge información de si la empresa ha introducido productos y/o servicios nuevos o mejorados al mercado antes que los compe- tidores, mientras que la segunda determina si la empresa ha introducido un producto o servicios mejorado de manera significativa, en función del que ya disponía en su mercado los competidores. La inclusión de innovaciones radicales e innovaciones incrementales, brinda la oportunidad de poder diferenciar a las empresas con una estructura clara de ACAP.

Para el caso de las variables independientes algunas de estas se describen como: localización y utilización de diferentes fuentes de información para las actividades de innovación, modificación y aplicación de nuevas formas organizativas y métodos de comercialización, identificación y aprovechamiento de factores internos y externos que motivan y facilitan las actividades de innovación, afectación de la actividad innovadora en los productos o servicios, procesos, medio ambiente y normativas de la organización y retribuciones e incentivos al personal dedicado a I+D, entre otras (Cuadro 2).

\section{Estructura de la ACAP en el sector servicios y su poder discriminante}

De los resultados del análisis factorial, se obtienen la conformación de 6 factores que identifica la estructura de los determinantes de la PCAP y la RACAP en el sector servicios. Los factores rotados que se presentan en la Tabla 1, explican de manera conjunta el $67 \%$ de la varianza total. El resultado del estadístico KaiserMeyer-Olkin (KMO) y la prueba de esfericidad de Bartlett son estadísticamente significativos, lo cual denota una buena robustez del modelo y, se evidencia un 


\section{Tabla 1 \\ Análisis factorial de componentes principales de ACAP (Rotación por Varimax)}

\begin{tabular}{|c|c|c|c|c|c|c|c|}
\hline \multirow[t]{2}{*}{ Indicadores } & \multirow{2}{*}{$\begin{array}{l}\text { Comuna- } \\
\text { lidades }\end{array}$} & \multicolumn{6}{|c|}{ Factores Rotados } \\
\hline & & 1 & 2 & 3 & 4 & 5 & 6 \\
\hline Innovación en servicios & .606 & & & 0,707 & & & \\
\hline Innovación en bienes & .603 & & & 0,709 & & & \\
\hline Innovación en productos & .696 & & & 0,725 & & & \\
\hline Innovaciones en nuevos métodos de comercialización & .559 & & & 0,680 & & & \\
\hline Innovaciones en las relaciones con otras empresas & .574 & & & & & & 0,700 \\
\hline $\begin{array}{l}\text { Innovaciones en las estructuras de gestión e integración de los departa- } \\
\text { mentos }\end{array}$ & .704 & & & & & & 0,817 \\
\hline Innovaciones en los sistemas de gestión de los conocimientos & .596 & & & & & & 0,700 \\
\hline Importancia del efecto sobre el cumplimiento de los requisitos normativos & .741 & & & & & 0,711 & \\
\hline Importancia del efecto sobre el impacto en el medio ambiente & .755 & & & & & 0,728 & \\
\hline Importancia del efecto sobre el impacto salud y seguridad & .713 & & 0,618 & & & & \\
\hline Importancia del efecto sobre los materiales por unidad producida & .783 & & 0,763 & & & & \\
\hline Importancia del efecto sobre la energía por unidad producida & .785 & & 0,773 & & & & \\
\hline Importancia del efecto sobre los costos laborales por unidad producida & .731 & & 0,727 & & & & \\
\hline $\begin{array}{l}\text { Importancia del efecto sobre la capacidad de producción o presta- } \\
\text { ción de servicios }\end{array}$ & .758 & & 0,718 & & & & \\
\hline $\begin{array}{l}\text { Importancia del efecto sobre la flexibilidad en la producción o la pres- } \\
\text { tación de servicios }\end{array}$ & .663 & 0,592 & & & & & \\
\hline Importancia del efecto sobre la calidad de los productos y servicios & .726 & 0,745 & & & & & \\
\hline Importancia del efecto sobre la cuota de mercado & .690 & 0,736 & & & & & \\
\hline Importancia del efecto sobre la penetración de nuevos mercados & .735 & 0,680 & & & & & \\
\hline Importancia del efecto sobre la sustitución de productos y servicios & 621 & 0,646 & & & & & \\
\hline $\begin{array}{l}\text { Importancia del efecto de la innovación sobre una gama más am- } \\
\text { plia de productos y/o servicios }\end{array}$ & .667 & 0,669 & & & & & \\
\hline $\begin{array}{l}\text { Incentivos y retribuciones a investigadores, técnicos auxiliares y/o } \\
\text { becarios }\end{array}$ & .627 & $-0,620$ & & & & & \\
\hline Fuentes Externas Institucionales de Información: Centros tecnológicos. & .485 & & & & 0,542 & & \\
\hline $\begin{array}{l}\text { Fuentes Externas de Información (Institucionales): Universidades } \\
\text { u otros centros de enseñanza superior }\end{array}$ & .671 & & & & 0,786 & & \\
\hline $\begin{array}{l}\text { Fuentes Externas de Información (Comerciales): consultores, labo- } \\
\text { ratorios comerciales privados de I+D }\end{array}$ & .725 & & & & 0,824 & & \\
\hline \multirow{2}{*}{$\begin{array}{l}\text { Fuentes Externas de Información (Mercado): consultores, labora- } \\
\text { torios comerciales privados de I+D } \\
\qquad \% \text { de varianza explicada }\end{array}$} & .633 & & & & 0,717 & & \\
\hline & & 38.4 & 7.74 & 6.54 & 5.79 & 4.51 & 4.30 \\
\hline \multicolumn{2}{|l|}{$\%$ Total de varianza explicada } & \multicolumn{6}{|c|}{67.34} \\
\hline \multicolumn{2}{|l|}{ MASA de Kaiser-Meyer-Olkin. } & \multicolumn{6}{|c|}{.916} \\
\hline Prueba de esfericidad de Bartlett (Sig) & & \multicolumn{6}{|c|}{.00} \\
\hline
\end{tabular}

${ }^{*} \mathrm{p}<.001,{ }^{* *} \mathrm{p}<.005$

Fuente: Elaboración propia. 
buen ajuste entre los factores y los datos. La comunalidad para todos los indicadores es superior a 0,5 , que junto al criterio de rotación por Varimax, garantiza una adecuada carga factorial en cada uno de los indicadores.

En definitiva, los resultados del análisis factorial evidencian la existencia de una estructura de ACAP para el sector servicio, cuantificable y moderadamente aproximada al modelo teórico de Zahra y George (2002). La estructura latente de ACAP en el sector servicios se muestra en el Cuadro 3 $y$ hace referencia a las actividades que potencian la PACAP y la RACAP.

En cuanto a la PACAP, las actividades de identificación de fuentes externas de conocimiento (Factor 4) son un proxy de la adquisición y asimilación del conocimiento; y engloba aquellas actividades relacionadas con fuentes de informaciones provenientes del mercado como: clientes, competidores y consultoras; fuentes de información institucionales como: universidades, organismos públicos de inversión y centros tecnológicos y otras fuentes como: conferencias, ferias comerciales y exposiciones. Estas actividades le permite a la empresa identificar el conocimiento existente en el exterior (Cohen y Levinthal, 1990); además de permitirle identificar y adquirir conocimiento externo, que por lo general es un elemento crítico para sus operaciones (Zahra y George, 2002). Así mismo, este factor está asociado con aquellas actividades que involucran la interpretación del conocimiento adquirido, la comprensión del entorno de la empresa, y la incorporación de recursos externos que favorecen los procesos de innovación (Kim, 1999).

Por otro lado, las actividades que estimulan la RACPA en las empresas del sector servicios como: las actividades de implementación, aplicación, conversión y re-codificación del esfuerzo innovador y el conocimiento; la introducción de nuevos o mejorados productos $\mathrm{y} / \mathrm{o}$ servicios $\mathrm{y}$ la combinación e interiorización del conocimiento base, adquirido $\mathrm{y} / \mathrm{o}$ asimilado (Factor 1, 2, 3, 5, y 6,) explican la transformación y explotación del conocimiento.

\section{Cuadro 3}

\section{Estructura de ACAP: factores explicativos en empresas de servicios}

$\begin{array}{ll}\text { PACAP } & \text { Factor 4: Identificación de fuentes externas de conocimiento (entorno } \\ & \text { directo de la organización, mercado e instituciones académicas). } \\ \text { RACAP } & \text { Factor 1: Implementación y aplicación del esfuerzo innovador sobre } \\ & \text { los productos y procesos. } \\ & \text { - Factor 2: Conversión y re-codificación del esfuerzo innovador y el co- } \\ & \text { nocimiento sobre los recursos y las operaciones. } \\ & \text { Factor 3: Introducción de nuevos o mejorados productos y/o servicios, } \\ & \text { resultantes del esfuerzo innovador. } \\ & \text { Factor 5: Implementación y aplicación del esfuerzo innovador sobre } \\ & \text { los otros factores organizacionales. } \\ & \text { Factor 6: Combinación e Interiorización del conocimiento base, adqui- } \\ & \text { rido y/o asimilado. }\end{array}$

Fuente: Elaboración propia. 
Estas dimensiones pueden ser descritas como todas aquellas actividades de refinación, extensión y apalancamiento de las rutinas organizaciones que conllevan a explotar el potencial y las capacidades de la empresa (Zahra y George, 2002). Tanto la realización de trabajos creativos dentro de la empresa que resultan en un aumento del volumen de conocimiento, como la introducción de productos nuevos o mejorados, mejoras en el nivel de calidad de los productos obtenidos, mayor preocupación por el nivel de impacto de los productos sobre el medioambiente, la salud y la seguridad; proveen a las empresas de capacidades que le permitan transformar y explotar el conocimiento con el fin de incrementar su resultado innovador.

Las puntuaciones factoriales obtenidas del análisis de componentes principales para la ACAP, permitieron la estimación de modelos discriminantes diferenciando obviar en términos del gasto en innovación (entradas) y de innovaciones radicales e innovaciones incrementales (salidas).

En la Tabla 2 se presenta el modelo discriminante para el gasto en innovación (Modelo 0). Los estadísticos M de box y Lambda de Wilks dan cuenta de la validez significativa del modelo, donde más del $89 \%$ de los casos fueron clasificados correctamente, lo que denota el poder predictivo de la función discriminante. Los resultados demuestran que existen diferencias significativas entre las empresas del sector servicios y se acepta la Hipótesis 1 ; de que la estructura de factores multivariantes de la PACAP y la RACAP, determina las diferencias en el gasto de innovación y el perfil entre las empresas del sector servicios. Pero más allá de este hallazgo, es posible deducir que al menos en las empresas del sector servicios la obtención de ACAP no depende del gasto de innovación previo que se realice.

Al comparar los coeficientes del modelo con las medias de los grupos (empresas que no realizaron gasto en innovación y empresas que realizaron gasto en innovación) se observa que las empresas que no realizaron gasto en innovación aprovechan más factores de ACAP (p.e. Factor 1: coef $=.529$, medias grupos $.399>-.567$. Factor 4: coef $=.367$, medias grupo $=.295>-.419)$, potenciando con ello tanto a la PACAP como a la RACAP; mientras que las empresas que realizan gasto en innovación aprovechan menos factores de ACAP, centrándose más en las RACAP (p.e. Factor 3:coef $=-.351$, medias grupos $-.284<.403$ ).

En el caso de las empresas que no realizan gasto en innovación los factores que más discriminan son los relacionados a las actividades de identificación de fuentes externas de conocimiento (Factor 4) y las actividades de implementación y aplicación del esfuerzo innovador sobre los productos y procesos (Factor 1). También se puede observar como las actividades de conversión y re-codificación del esfuerzo innovador y el conocimiento sobre los recursos y las operaciones (Factor 2) y las actividades de implementación y aplicación del esfuerzo innovador sobre los otros factores organizacionales (Factor 5) resultan significativas y discriminantes, aunque con un menor poder explicativo, para este grupo de empresas. En la Tabla 2, las medias de los factores discriminantes de estas empresas es mayor que las medias de las empresas que realizan gasto en innovación. 


\section{Tabla 2}

\section{Análisis discriminante. Matriz de estructura} Entradas del Procesos de Innovación: Gasto de Innovación

\begin{tabular}{|c|c|c|c|c|}
\hline Dimensión & Factor & $\begin{array}{l}\text { Modelo } \\
0\end{array}$ & $\begin{array}{c}\text { Media } \\
\text { Grupo } \\
\text { Sin Gasto } \\
\text { Innovación } \\
\text { (obs=2292) }\end{array}$ & $\begin{array}{c}\text { Media } \\
\text { Grupo } \\
\text { Con Gasto } \\
\text { Innovación } \\
\text { (obs }=2107 \text { ) }\end{array}$ \\
\hline \multirow[t]{2}{*}{ PACAP } & $\begin{array}{l}\text { Factor 4: Identificación de Fuentes Externas de } \\
\text { Conocimiento (entorno directo de la organiza- } \\
\text { ción, mercado e instituciones académicas). }\end{array}$ & $.367^{*}$ & .295 & -.419 \\
\hline & $\begin{array}{l}\text { Factor 2: Conversión y re-codificación del es- } \\
\text { fuerzo innovador y el conocimiento sobre los } \\
\text { recursos y las operaciones. }\end{array}$ & $.130^{*}$ & .110 & -.156 \\
\hline \multirow[t]{8}{*}{ RACAP } & $\begin{array}{l}\text { Factor 6: Combinación e Interiorización del } \\
\text { conocimiento base, adquirido y/o asimilado. }\end{array}$ & $-.96^{\star}$ & -.081 & .116 \\
\hline & $\begin{array}{l}\text { Factor 5: Implementación y aplicación del es- } \\
\text { fuerzo innovador sobre los otros factores or- } \\
\text { ganizacionales }\end{array}$ & $.138^{*}$ & .117 & -.167 \\
\hline & $\begin{array}{l}\text { Factor 1: Implementación y aplicación del es- } \\
\text { fuerzo innovador sobre los productos y pro- } \\
\text { cesos. }\end{array}$ & $.529^{\star}$ & .399 & -.567 \\
\hline & $\begin{array}{l}\text { Factor 3: Introducción de nuevos o mejora- } \\
\text { dos productos y/o servicios, resultantes del } \\
\text { esfuerzo innovador }\end{array}$ & $-.351^{*}$ & -.284 & .403 \\
\hline & M de box (sig): & .00 & & \\
\hline & Lambda de Wilks (sig): & .00 & & \\
\hline & Autovalor: & 1.049 & & \\
\hline & Correlación Canoníca: & .716 & & \\
\hline
\end{tabular}

${ }^{*} p<.001,{ }^{* *} p<.005$.

Fuente: Elaboración propia.

Las empresas que realizan gasto en innovación, se apoyan más en actividades relacionadas con la introducción de nuevos o mejorados productos $\mathrm{y} / \mathrm{o}$ servicios, resultantes del esfuerzo innovador y actividades de combinación e interiorización del conocimiento base adquirido y asimilado; actividades estas que potencian solo la RACAP. El hecho de invertir recursos financieros hace que las empresas busquen resultados con- cretos de innovación en producto y/o servicios. Cuando las empresas del sector servicios hacen inversiones en innovación dan menos importancia a factores relacionados con la adquisición y asimilación del conocimiento (PACAP), que a factores relacionados con la RACAP; de hecho demuestran un especial interés por las actividades de explotación del conocimiento frente a las actividades de transformación. 
En la Tabla 3 se presentan los resultados para el caso de los modelos discriminantes para innovaciones radicales (Modelo 1) y para innovaciones incrementales (Modelo 2), ambos salidas del proceso innovador. Los estadísticos $\mathrm{M}$ de box y Lambda de Wilks dan cuenta de la validez significativa de estos dos modelos. En el caso de las empresas que obtuvieron innovaciones radicales, el $74 \%$ de los casos fueron clasificados correctamente y un $82 \%$ en el caso de las innovaciones incrementales, lo que denota el poder predictivo de las funciones discriminantes. Para ambos modelos, los resultados obtenidos confirman la Hipótesis 2 de que la estructura de factores multivariantes de la PACAP y RACAP, determinan las diferencias en los resultados innovadores y el perfil entre las empresas del sector servicios.

Siguiendo con el análisis de la Tabla 3, para el caso de las empresas del sector servicios que obtuvieron innovaciones radicales los resultados del estudio demostraron que dichas empresas se aprovechan más de factores de ACAP asociados a la RACAP. Esto quiere decir que las empresas del sector servicios con resultados novedosos potencian sus actividades de explotación del conocimiento, con especial énfasis en las actividades dirigidas a introducir nuevos productos, aplicar el esfuerzo innovador sobre los productos y procesos, y convertir o re-codificar el esfuerzo innovador y el conocimiento sobre los recursos y las operaciones (Zahra y George, 2002). Por el contrario, las empresas que no realizaron in- novaciones radicales son empresas que dieron énfasis tanto a la PACAP como a la RACAP. Sin embargo, a pesar de no haber obtenido resultados con alto grado de novedad, hay una importante tendencia de las empresas por aprovechar factores de ACAP relacionados con la explotación del conocimiento.

De acuerdo con lo anterior, una empresa del sector servicios que quiera obtener innovaciones radicales deberá centrar su atención en promover objetivos de innovación orientados a mejorar aspectos como: la flexibilidad en su capacidad de producción o prestación del servicio, el control sobre los costos de mano de obra y materia prima con especial tendencia a su disminución y la promoción de actividades orientadas a la creación de nuevos bienes y servicios.

En el caso de las empresas que obtuvieron innovaciones incrementales (Tabla 3 ), los resultados del estudio son opuestos. Se observa que para este tipo de innovaciones las empresas se han aprovechado de actividades relacionadas con la RACAP. Es decir, concretamente actividades relacionadas con la introducción al mercado de productos y/o servicios mejorados (Factor 3 ) y actividades relacionadas con la combinación e interiorización del conocimiento base adquirido y asimilado (Factor 6). Por el contrario las empresas que no obtuvieron innovaciones incrementales, se apalancaron tanto en la PACAP como en la RACAP, con el especial énfasis en actividades orientadas a la explotación del conocimiento. 


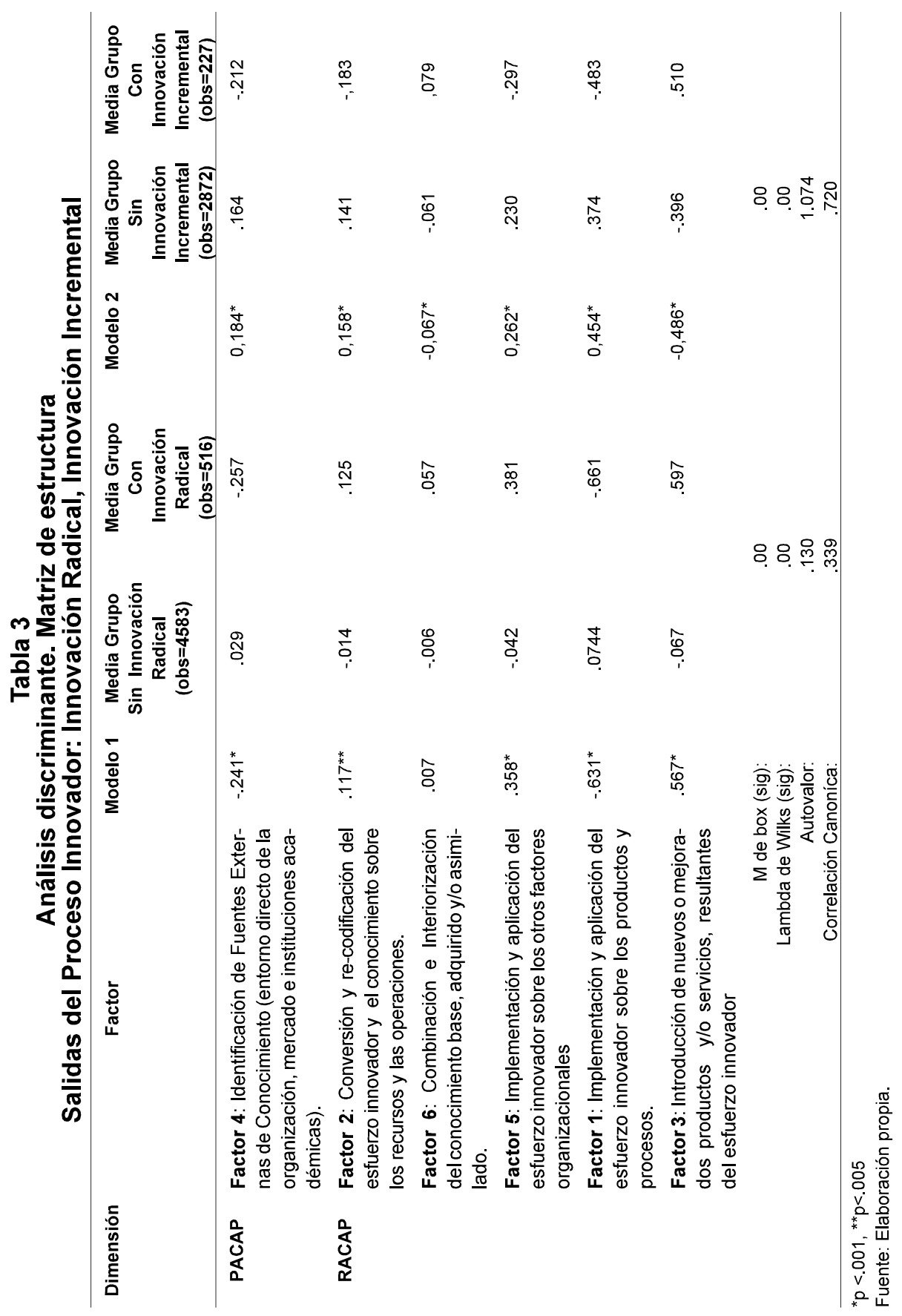




\section{Conclusiones}

La forma como se comportan las empresas del sector servicios entre sí, es muy particular. Estudios e investigaciones previas demuestran que la actividad innovadora en cada empresa es compleja y única a la vez, involucrando muchos factores que en ocasiones no se comportan de manera similar. Cuando las empresas realizan gasto en innovación u obtienen algún resultado innovador demuestran un claro interés por potenciar o aprovecharse de una parte del proceso de ACAP, la RACAP. Pero cuando no realizan gasto en innovación o no obtienen resultado innovador alguno, prestan una mayor atención a la PACAP y a la RACAP, es decir toman en su conjunto todo el proceso de ACAP. Esto parece indicar que encuentran en la ACAP una oportunidad para obtener ventajas competitivas; así como lograr cierta diferenciación entre sí. El reconocimiento explícito de la relación entre estos dos componentes explica por qué las empresas son capaces de aprovechar y aplicar nuevo conocimiento.

Tradicionalmente, unos de los parámetros más utilizados para medir ACAP ha sido el gasto en innovación; no obstante ésta es una medida de utilidad limitada para estimar dicha variable. Al observar la conducta de las empresas que realizan gasto en innovación frente a las que no, queda claro que el gasto en innovación no es siempre en sí misma una variable que indique la presencia de ACAP, y más aún no es una medida adecuada para explicar de forma total la dinámica de la innovación en el sector servicios.
Ampliamente se sabe que la cuantía de los resultados, en términos relativos; en el sector servicios es mucho menor que en otros sectores de actividad; sin embargo el peso que tiene en el desarrollo económico hace que sus resultados de innovación sean importantes y relevantes, en términos absolutos. De manera más detallada, el estudio concluye que al observar la distribución de las empresas del sector servicios en función del resultado innovador, se percibe una clara tendencia a la innovación incremental, lo que refleja que la innovación en servicios tiene una orientación mayoritaria al desarrollo de cambios organizativos y de procesos, y presenta un menor interés por la introducción de nuevos productos al mercado. Esto le otorga una mayor flexibilidad en su modelo de producción o prestación del servicio, y por tanto le permite una mejor adecuación a las necesidades cambiantes del mercado.

Los resultados de este estudio confirman que para las empresas del sector servicios en España, existe una tendencia clara y definida a reconocer la importancia de adquirir, asimilar, transformar y explotar el conocimiento. También se puede reconocer que incorporan cada vez más el valor de la innovación en sus operaciones, con lo cual exhiben de manera significativa una ventaja que las deferencias en términos de entradas y salidas del proceso innovador.

Otra conclusión que puede ser establecida es que partiendo de la revisión de la literatura y la concienzuda operacionalización de ciertos enfoques de estrategia, competitividad e innovación, ha sido viable conformar un marco referencial 
que ayude, de manera cuantitativa, al estudio y compresión del impacto de ACAP sobre el desempeño de las rutinas organizativas en el sector servicios y su resultado innovador. Con referencia a la presencia significativa de ACAP en las empresas estudiadas, aquellas que contaban con una base tecnológica existente 0 realizaron algún esfuerzo en la entrada del proceso innovador, alcanzaron un determinado nivel de PACAP y RACAP, que les llevo a obtener resultados innovadores; lo cual podría derivar en ventajas competitivas capaces de ser sostenidas en el tiempo y con efectos favorables sobre sus resultados económicos.

La estructura de ACAP obtenida, con naturaleza fundamentalmente cuantitativa, para el sector servicio y la confirmación del impacto positivo de dicha estructura sobre el resultado innovador, ofrece una mayor apertura en el estudio de este campo. Los hallazgos obtenidos podrían sentar las bases para el estudio de la capacidad de absorción especializada en este sector. Una capacidad de absorción especializada en el sector servicios, en nuestro mejor conocimiento, puede ser entendida como una habilidad de las organizaciones, que partiendo de la existencia de conocimiento tácito, pueda reconocer e interiorizar más directamente el conocimiento externo, aplicándolo para generar valor a los consumidores y al mercado.

Este trabajo tiene algunas limitaciones que sugieren posibilidades para futuras líneas de investigación. El estudios es de corte transversal y futuras investigaciones deberían explorar la interacción de las variables en el tiempo para determinar si la estructura de la ACAP cambia con el avance tecnológico o los cambios económicos. Adicionalmente sería interesante establecer la importancia relativa de cada factor o componente de la ACAP, para conocer si hay diferencias significativas con el sector manufacturero, donde el concepto ha sido ampliamente contrastado.

\section{Referencias Bibliográficas}

Amara, Nabil; Rajean Landry y David Doloreux (2009). Patterns of Innovation in Knowledge-Intensive Business Services. The Service Industries Journal, Vol. 29, No. 4, pp. 407-430.

Arbussa, Anna y Coenders Germá (2007). Innovation Activities, use of Appropriation Instruments and Absorptive Capacity: Evidence from Spanish Firms. Research Policy, Vol.36, No.10, pp. 1545-1558.

Caloghirou, Yannis; Kastelli loanna y Tsakanikas Aggelos (2004). Internal Capabilities and External Knowledge Sources: Complements or Substitutes for Innovative Performance? Technovation, Vol. 24, No.1, pp. 29-39.

Chen, Chung-Jen (2004). The Effect of Knowledge Attribute, Alliance Characteristics, and Absorptive Capacity on Knowledge Transfer Performance. R \& D Management, Vol. 34, No. 3, pp. 311-321.

Cohen, Wesley M. y Levinthal, Daniel A. (1990). Absorptive Capacity: A New Perspective on Learning and Innovation. Administrative Science Quarterly, Vol. 35, No. 1, pp.128-152.

Cohen, Wesley M. y Levinthal, Daniel A. (1989). Innovation and Learning: The Two Faces of R\&D. The Economic Journal, Vol. 99, No. 397, pp. 569-596. 
Di Stefano Giada; Peteraf, Margaret y Verona Gianmario (2010). Dynamic Capabilities Deconstructed: A Bibliographic Investigation into the Origins, Development, and Future Directions of the Research Domain. Industrial and Corporate Change, Vol. 19, No. 4, pp. 1187-1204.

Dunning, John y Lundan Sarianna (2010). The Institutional Origins of Dynamic Capabilities in Multinational Enterprises. Industrial and Corporate Change, Vol. 19, No. 4, pp. 122-1246.

Escribano, Alvaro; Fosfuri, Andrea y Tribó Josep A. (2009). Managing External Knowledge Flows: The Moderating Role of Absorptive Capacity. Research Policy, Vol. 38, No.1, pp. 96-105.

Hair, Jeffre. Tatham, Ronald (1999). Análisis Multivariante. Ed. Prentice Hall. Londres.

Jansen, Justin; Van Den Bosch, Frans. A. J. y Volberda Henk. W. (2005). Managing Potential and Realized Absorptive Capacity: How do Organizational Antecedents Matter? Elsevier, Vol. 36, No. 10 , pp. 1545-1558.

Jantunen, Ari; Puumalainen, Kaisu; Saarenketo, Sami; Kyläheiko, Kalevi (2005). Entrepreneurial orientation, dynamic capabilities and international performance. Journal of International Entrepreneurship, Vol. 3, No. 3, pp. 223-243.

Jiménez-Barrionuevo, María. M.; GarcíaMorales Victor. J. y Molina Luis. M. (2011). Validation of an Instrument to Measure Absorptive Capacity.Technovation, Vol.35, No. 5, pp.190-202.

Khoja, Faiza y Maranville, Steven (2010). How do Firms Nurture Absorptive Capacity? Journal of Managerial Issues, Vol. 22, No. 2, pp. 262-361.

Kim, Linsu (1980). Stages of Development of Industrial Technology in a Developing
Country: A Model. Research Policy, Vol. 9, No. 3, pp. 254-277.

Kim, Lindsay (1999). Building Technological Capability for Industrialization: Analytical Frameworks and Korea's Experience. Industrial and Corporate Change, Vol. 8, No. 1, pp.111-136.

Kostopoulos, Konstatinos; Papalexandris, Alexandro; Papachroni, M. y loannou, G. (2011). Absorptive Capacity, Innovation, and Financial Performance. Journal of Business Research, Vol. 36, No. 10, pp. 1545-1558.

Lane, Peter J. y Lubatkin, Michael (1998). Relative Absorptive Capacity and Inter-organizational Learning. Strategic Management Journal, Vol. 19, No. 5, pp. 461-477.

Miles, Ian; Kastrinos, Nikos; Flanagan, Kieron; Bilderbeek, Rob; Den Hertog, Pim; Huntink, Willen; Bouman, Mark (1995). Knowledge-intensive business services. EIMS Publication, U.K.

Mowery, David C.; Oxley, Joanne E. y Silverman, Brian S. (1996). Strategic Alliances and Inter-firm Knowledge Transfer. Strategic Management Journal, Vol. 17, No. 1, pp. 77-91.

Murovec, Nika y Prodan, Igor (2009). Absorptive Capacity, its Determinants, and Influence on Innovation Output: Cross-Cultural Validation of the Structural Model. Technovation, Vol. 29, No. 12 , pp. 859-872.

Narasimhan, Om; Rajiv, Surendra y Dutta, Shantanu (2006). Absorptive Capacity in High-Technology Markets: The Competitive Advantage of the Haves. Marketing Science, Vol. 25, No. 5, pp. 510-522.

Newey, Lance R. y Shulman, Arthur D. (2004). Systemic Absorptive Capacity: Creating Early-to-Market Returns through R\&D Alliances. R \& D Management, Vol. 34, No. 5, pp. 495-504. 
Nieto, Mariano y Quevedo, Pilar (2005). Absorptive Capacity, Technological Opportunity, Knowledge Spillovers, and Innovative Effort. Technovation, Vol. 25 , No. 10 , pp. $1141-1157$.

Ortega, María (2010). Competitive Strategies and Firm Performance: Technological Capabilities' Moderating Roles. Journal of Business Research, Vol. 63, No. 12, pp. 1273-1281.

Rush, Howard; Bessant, John y Hobday, Mike (2007). Assessing the Technological Capabilities of Firms: Developing a Policy Tool. R\&D Management, Vol. 37, No. 3, pp. 221-236.

Szulanski, Gabriel (1996). Exploring Internal Stickiness: Impediments to the Transfer of Best Practice within the Firm. Strategic Management Journal, Vol. 17, pp. 27-43.

Teece, David J.; Pisano, Gary y Shuen, Amy (1997). Dynamic Capabilities and Strategic Management. Strategic Management Journal, Vol. 18, No.7, pp. 509.

Tether, Bruce S. y Tajar, A. (2008). Beyond Industry-University Links: Sourcing Knowledge for Innovation from Consultants, Private Research Organizations and the Public Science-Base. Research Policy, Vol. 37, No. 6-7, pp. 1079-1095.

Todorova, Gergana y Durisin, Boris (2007). Absorptive Capacity: Valuing a Reconceptualization. The Academy of Management Review, Vol. 32, No. 3, pp. 774-786.
Tsai, Kun Hsien (2009). (Collaborative Networks and Product Innovation Performance: Toward a Contingency Perspective. Research Policy, Vol. 38, No. 5, pp. 765-778.

Van den Bosch, Frans; Volberda, Henk W. y De Boer, Michiel (1999). Coevolution of Firm Absorptive Capacity and Knowledge Environment: Organizational Forms and Combinative Capabilities. Organization Science, Vol. 10, No. 5, pp. 551-568.

Volberda, Henk; Foss, Nicolai y Lyles, Marjorie (2010). Absorbing the Concept of Absorptive Capacity: How to Realize its Potential in the Organization Field. Organization Science, Vol. 21, No. 4, pp. 931-951.

Zahra, Shaker A.; Sapienza, Harry J. y Davidsson, Per (2006). Entrepreneurship and Dynamic Capabilities: A Review, Model and Research Agenda. Journal of Management Studies, Vol. 43, No. 4, pp. 917-955.

Zahra, Shaker A. y George, Gerard (2002). Absorptive Capacity: A Review, Reconceptualization, and Extension. Academy of Management. The Academy of Management Review, Vol. 27, No. 2, pp.185-203.

Ziggers, George.W. y Joshep. Henseler. (2009). Inter-Firm Network Capability: How it Affects Buyer-Supplier Performance. British Food Journal, Vol. 111, No. 8, pp. 794-810. 